and pharmacy benefit mangers, that is, the adequacy of pharmacy benefits and the potential consequences that could arise from gaps in treatment associated with financial barriers. A Kaiser Family Foundation study published in 2002 found that 30\% of the nonelderly uninsured did not fill a prescription in the previous 12 months due to cost, compared to $12 \%$ of the nonelderly with insurance, ${ }^{26}$ and $10 \%$ of the insured population reported needing a prescription in the previous 12 months but not getting it due to cost. ${ }^{27}$ Overall, nearly one quarter of seniors, regardless of drug benefit coverage, either did not fill a prescription or skipped doses due to costs. More than one third of seniors without drug coverage either skipped doses or did not fill a prescription due to costs, twice the rate of seniors with drug benefit coverage. ${ }^{24}$

Perhaps the most important finding by Cox and Henderson is the apparent link between Medicare prescription drug benefit maximums and the use of prescription drug samples as a means to mitigate out-of-pocket expenditures. This practice is potentially self-defeating since (a) higher-cost drugs are more heavily sampled and (b) the availability of drug samples may affect physician prescribing practices and reduce the pressure to find lower-cost therapeutic alternatives. Other investigators may ask Medicare+Choice beneficiaries with drug benefit limits the important questions regarding the nature and usefulness of interactions with physicians in offering recommendations for generic drugs and other lower-cost therapeutic alternatives to help reduce out-of-pocket expenditures.

\section{Crossing the Quality Chasm- Pharmacist Prescribing, Nontraditional Interventions, and Outcomes-based Pharmacist Reimbursement (OBPR)}

Since 1996, pharmacists in Indiana have had the authority to manage the drug therapy of hospital patients, including prescribing drugs, when conducted via a protocol established with a physician and a hospital. ${ }^{28}$ In California, AB 826 was enacted late in 2001 and became effective January 1, 2002. AB 826 included 2 key changes in California law: (1) pharmacists may perform clinical functions outside of a pharmacy or other licensed health facility (e.g., the patient's home, physician's office, or medical office building) and (2) pharmacists may select initial drug therapy. Prior law only allowed adjustment of drug therapy (e.g., dose) in outpatient settings. Pharmacist prescriptive authority is permitted only under appropriate protocols, and the pharmacist must notify the prescriber within 24 hours of initiating a drug regimen under this new authority. ${ }^{29}$

By 2002, 33 states granted prescriptive authority to pharmacists, ${ }^{30}$ typically permitting pharmacists to work collaboratively with physicians to adjust drug therapy under protocol. ${ }^{31}$ The collaborative practice agreement with a physician(s) may specify authority for the pharmacist to adjust the dose of prescribed drugs, or in many situations, select initial drug therapy as well. In 1999, Minnesota pharmacists could establish collab- orative practice agreements with physicians allowing pharmacists to adjust drug therapy and administer the first dose in an emergency. The amendments to the pharmacy practice act also recognized the pharmacist's role in counseling, monitoring, and drug research. ${ }^{32}$ In 2002, Maryland HB781 allowed a licensed physician and a licensed pharmacist to enter into a drug therapy management contract. ${ }^{33}$ As defined in the Maryland bill, the contract is a voluntary written arrangement that is disease-state-specific and applies to one pharmacist, one physician, and one patient receiving care from the physician and pharmacist. Connecticut SB528, also enacted in 2002, allowed one or more licensed pharmacists employed by a hospital to enter into a written protocolbased collaborative drug therapy management agreement with one or more physicians to manage the drug therapy of individual patients receiving inpatient services in a hospital. ${ }^{34}$

The need for collaborative work among physicians and pharmacists to reduce medication errors is well-recognized in health systems devoted to quality improvement principles. These organizations are more likely to spawn solutions that recognize the strengths of pharmacists in knowledge of drug dosing and even drug selection. At the University of Wisconsin Hospitals and Clinics, pharmacists have (veto) authority over the prescribing of physicians, most of whom on the house staff are interns and residents. A trial program begun in the trauma unit in 1996 was expanded to a hospital-wide program in 2002, first for all anti-infective orders and later for other therapeutic categories. ${ }^{35}$ Pharmacist oversight of drug orders at the University of Wisconsin Hospitals and Clinics is directed by the hospital's pharmacy and therapeutics committee and will be supplemented in the future by information technology, a computerized physician order entry system.

Recent reports of prescriptive authority for pharmacists and clinical pharmacy interventions in nontraditional roles include a telephone clinic and protocol for eradication of Helicobacter pylori infection. The intervention was associated with favorable outcomes: (a) 100\% patient compliance with HP eradication therapy, (b) symptom improvement in 33\% of patients (but many patients were either asymptomatic or mildly symptomatic before initiating HP eradication therapy), (c) no drug-drug or drug-food interactions, (d) no reinitiation of acid-suppressive therapy at one-month post-treatment, (e) creation of a learning environment for doctor of pharmacy clerkship students in defining a new role for pharmacists in ambulatory care, and (f) pharmacy collaboration with the departments of surgery, medicine, and nursing. ${ }^{36}$

The entire process of quality improvement in health care is based upon incremental change. While the Leapfrog Group encourages us to think of dramatic, fundamental change, the reality is less cataclysmic. As with most industrial and engineering processes, improving the tools improves the quality of the product but only with changes in processes by which the 
tools are used. In drug therapy management, we need the operators (pharmacists) of the tools to embrace clinical practice guidelines and the principles of continuous quality improvement. Compensating pharmacists at the lowest possible price does not foster this attention and commitment.

Farris, Kumbera, Halterman, and Fang in this issue of the Journal, describe a proprietary program developed around the concept of compensating pharmacists based upon measurable patient outcomes. ${ }^{37}$ This is the holy grail of managed care pharmacy, compensation for clinical pharmacists based upon valued outcomes.

\section{Statistical Significance Versus Practical Significance}

Researchers know the value of sample size in demonstrating statistical significance between groups. Small sample size requires a large absolute difference between groups to demonstrate statistical significance. For example, the data used to obtain FDA approval of pravastatin for the indication of stroke involved a study of 9,014 patients with a history of myocardial infarction or unstable angina and total cholesterol levels of 155 to $271 \mathrm{mg}$ per deciliter. The study found that the risk of stroke was 3.7\% among patients given pravastatin versus $4.5 \%$ among patients given placebo over a follow-up period of 6 years, an absolute difference of $0.8 \%$ and relative risk reduction for stroke of $19 \% .{ }^{38}$

Subsequent scrutiny of the study findings and the literature citations identified flaws. Of the 4 studies cited by the authors to support the reported reductions of $25 \%$ to $30 \%$ in the rate of stroke, the West of Scotland trial showed no reduction in the rate of stroke-the risk after 5 years of follow-up was reported as 1.6 percent for both the pravastatin and placebo groups $(P=0.57)$, and a second cited study (the Air Force-Texas Coronary Atherosclerosis Prevention Study, or AFCAPS/ TexCAPS) did not list stroke as an individual end point. More to the point here, the 0.05 statistical difference reported by the authors using univariate analysis for pravastatin versus placebo was actually 0.10 upon application of multivariate analysis, arguably the more appropriate measure. ${ }^{39}$ Aside from the statistical debate, in which a small absolute difference may or may not be statistically "significant," there is the very important matter of practical significance. The study results suggested that it would require 750 patients to be treated for one year to prevent one nonfatal stroke, or more than $\$ 750,000$ in discounted drug cost, an unfavorable cost-effectiveness outcome and a very unfavorable cost outcome, compared to the risk reduction possible with aspirin. ${ }^{40}$ Previous research and a meta-analysis of clinical studies found no association between cholesterol levels and risk of stroke.

In this issue of the Journal, Arocho, Solis, Wade, Goldberg, and Tang discuss the very important subject of underlying characteristics of groups that might explain differences in the dependent measure found in study results. ${ }^{41}$ But within their analysis is the ever-present matter of practical versus statistical significance. As part of their findings of underlying differences among calcium channel blocker patients, they report $86 \%$ of amlodipine users had at least one professional service, compared to $84 \%$ of felodipine users $(P<0.05)$, in a study involving 17,667 patients. They also report a statistically significant $(P=0.0007)$ difference between average daily dose, $5.8 \mathrm{mg}$ for amlodipine patients versus $5.93 \mathrm{mg}$ for felodipine patients. They report average age but not median age and do not address the fact that $39.2 \%$ of amlodipine patients in their study were less than age 70 versus $33.9 \%$ of felodipine patients less than age 70 . Readers should note that this study was funded by the manufacturer of amlodipine. Managed care pharmacists would be interested in the formulary status of these two drugs at the time that the drug and medical claims were incurred in the health plans employed in the study, a fact not disclosed by the authors. Nevertheless, the authors are correct in highlighting the importance of managed care pharmacists investigating the underlying differences in patient populations, particularly in drug-todrug comparisons.

\section{Prevalence and Costs of Atopic Dermatitis}

Atopic dermatitis (AD), or eczema, is a chronic, relapsing inflammatory skin disease affecting about $10 \%$ of the Western population, with a prevalence estimate of $4 \%$ to $20 \%{ }^{42}$ The high range of the prevalence estimate is in children, and $80 \%$ to $90 \%$ of AD cases are diagnosed by age $5 .{ }^{43}$ The disease is physically distressing to the patient and often has an adverse effect on the quality of life. ${ }^{44}$ Fivenson, Arnold, Kaniecki, Cohen, Frech, and Finlay, in this issue of the Journal, provide estimates of some of the direct and indirect costs of $\mathrm{AD}$ among patients identified in one health plan, expressed in 1997 dollars ${ }^{45}$ Readers might note that about two thirds of the patients were cared for only by a dermatologist, suggesting that these patients were the more severe cases of $\mathrm{AD}$ in that population. Combined with the small prevalence, approximately $1.3 \%$ of plan members, the data suggest that this patient population is more representative of the moderate-to-severe population of $\mathrm{AD}$ patients. Yet, only 3 patients were rated as "severe" by provider-assessment. Nearly two thirds of the patients in this cohort were younger than 16 years, and $19.5 \%$ were younger than 4 years. There was one inpatient visit and no emergency room visits among 3,576 patientmonths in the study. The only inpatient visit occurred in a patient with a "mild" case of $\mathrm{AD}$, according to provider-assessed severity.

\section{בias Management-The Path to Better Evidence}

In this issue and on the Journal Web site (www.amcp.org) is a copy of the editorial policy, disclosure statement, and attestation required of all authors of articles published in JMCP, effective with the January/February 2002 issue. The detail is designed to obtain all disclosures necessary to permit readers to evaluate potential biases that may affect the objectives of the research, interpretation of data, conclusions, or other findings and to attribute to each listed author the specific contribution to the article.

Frederic R. Curtiss, PhD, RPh, CEBS, Editor-in-Chief 